\title{
Role of Ultrasonography in Screening High Risk Mothers for Detection of IUGR
}

\author{
Gattani Chandrashekhar $\mathbf{M}^{\mathbf{1}}$, Reddy Pradeep ${ }^{2}$ \\ ${ }^{1}$ Professor, Dept. of Radiodiagnosis, Mamata Medical College, Khammam \\ ${ }^{2}$ Junior Resident, Dept. of Radiodiagnosis, Mamata Medical College, Khammam
}

Corresponding Author: Gattani Chandrashekhar M

\begin{abstract}
Introduction: The 10th percentile of expected foetal weight for gestational age is classified as intrauterine growth restriction (IUGR) or foetal growth restriction. IUGR occurs for a variety of reasons. Any issue with the placenta is a major contributor.

Aims and objectives: 1) To determine the role of ultrasonography in screening high risk mothers for detection of IUGR.

2) To find out the impact of fetal parameters on the extent of IUGR.3) For correlation between sonographic pattern of IUGR and the birth weight. 4) For correlation between doppler parameters and perinatal outcome.
\end{abstract}

Materials and methods: This is a prospective study done over a period of 2 years in Mamata General Hospital (MGH) from Oct 2017 to Oct 2019. Study included 50 pregnant women with high risk factors and clinically suspected IUGR attending for ultrasound examination were subjected to Uterine, Umbilical and Middle cerebral artery Doppler along with morphology and biometry scan after fulfilling the inclusion and exclusion criteria.

Results: Reverse, absence or slow end diastolic flow was considered abnormal which was seen in 33 patients. Nine patients had Absent /Reversed end diastolic flow of which, 3 patients had IUD, 4 had Still births, 2 were admitted in NICU with Apgar at $1 \mathrm{~min}<7$. Middle cerebral artery was abnormal in 10 patients, of which 8 patients had IUGR babies with adverse perinatal outcome. MCA/UA S.D ratio, which was abnormal in 19 patients of which 16 patients had adverse perinatal outcome.

Conclusion: Doppler has proven to be more sensitive than other methods of foetal monitoring in recognising fetal compromises early and assisting in the proper time of delivery.

Keywords: Ultrasonography, doppler, IUGR.

\section{INTRODUCTION}

The 10th percentile of expected fetal weight for gestational age is classified as intrauterine growth restriction (IUGR) or fetal growth restriction1. It is a serious condition in which fetal development is insufficient and the baby is smaller than expected for its age.

IUGR is one of the most common causes of stillbirth, with over half of all newborns being underweight. The remainder of infants who survive severe IUGR have a higher risk of developmental delays, cerebral palsy, and neurobehavioral issues such as poor school performance, social skills, and fine motor control.

IUGR occurs for a variety of causes. Any issue with the placenta is a major contributor. Doppler imaging of the umbilical arteries can detect a reduction in placental vascular resistance and blood flow, which increases the risk of fetal acidosis2. Genetic abnormalities, congenital infections, maternal illnesses, and medications are some of the less prevalent reasons. 3

There are two forms of IUGR. Whether symmetrical or asymmetrical, symmetrical growth restriction is characterized by early beginning in the second trimester and a proportionate 
decrease in fetal indices. In fetuses with chromosomal abnormalities or infections, symmetrical IUGR is common. However, asymmetrical IUGR is characterized by late start and "brain sparing" at the expense of abdominal and soft tissue development, with various degrees of fetal length.

Doppler ultrasonography is often used in the assessment of pregnancies affected by intrauterine growth restriction to monitor blood flow via major fetal arteries or veins.

Because of a decrease in placental vascular resistance and an increase in enddiastolic velocity, which occurs physiologically, umbilical artery angle independent measures like pulsatility index or systolic/diastolic (S/D) ratio decrease with increasing gestational age. Due to blood shunting to critical organs (brain, heart), the EDF decreases in pathologic circumstances like IUGR foetuses, the umbilical artery wave shapes alter, and angle-independent indicators become aberrant with values above their reference ranges. The increased placental vascular resistance is reflected in these changes5. In this study we focus our measurements pulsatility index on fetal umbilical, middlecerebral arteries using Doppler velocimetry because of their importance for detection of any abnormalities underlying IUGR.

\section{MATERIALS AND METHODS}

This is a prospective study done over a period of 2 years in Mamata General Hospital (MGH) from Oct 2017 to Oct 2019. Study included 50 pregnant women with high risk factors and clinically suspected IUGR attending for ultrasound examination were subjected to Uterine, Umbilical and Middle cerebral artery Doppler along with morphology and biometry scan after fulfilling the inclusion and exclusion criteria.

The patients included in the study if the LMP (Last menstrual period) of the patient is well known, gestational age of patient between 13 and 41 weeks (from LMP), patient is a clinically diagnosed case of IUGR based on findings such as insufficient weight gain, decrease or no increase in abdominal girth, decrease or no increase in fundal height. If the patient was a know case of preeclampsia, maternal essential hypertension and previous IUGR.

Patients were excluded from the study if patient had multiple pregnancies, and congenital anomalies in the foetus.

Ultrasonography and colour Doppler were performed on "ESOATE MYLAB 60 , Aloka Prosound and Sonoscape "colour Doppler machine with curvilinear probe 1-8 MHz. With ultrasonography fetal biometry and morphology scan was done then Doppler was switched on. Patient is put in recumbent position with transducer in the longitudinal plane. The external iliac artery is visualised at pelvic side wall with colour Doppler. The transducer is then angled medially towards the uterine artery, where they cross the iliac artery. The flow velocity waveforms on the right and left uterine arteries then, when 3 or 4 waves of equal height were seen, the image was frozen and measurements were taken either by trace method/ manually/automatic trace. Then Doppler indices were obtained directly from the machine.

Then transducer is placed over anterior abdominal wall over the uterus and is carefully manipulated till a free loop of umbilical cord seen by Grey scale imaging and colour was used to identify the umbilical artery. Thus, Doppler waveform was obtained. Recordings of umbilical artery are obtained from free loop of umbilical cord. These were identified with the characteristic audio output and typical Doppler shift waveforms appearance on the screen. It was done in fetal apnea as breathing alters the Doppler shifts.

A transverse view of the fetal brain is obtained at the level of the biparietal diameter. The transducer is then moved towards the base of the skull at the level of the lesser wing of the sphenoid bone. Using color flow imaging, the middle cerebral artery is a major lateral branch of the circle of Willis, running anterolaterally at the 
borderline between the anterior and the middle cerebral fossae. The pulsed Doppler sample gate is then placed on the middle portion of this vessel to obtain flow velocity waveforms.

Parameters studied were S/D ratio and PI in Umbilical artery, S/D ratio in uterine artery and PI in Middle cerebral artery. Early diastolic notch in uterine artery, Absent and Reverse diastolic flow in umbilical artery were watched.

The flow velocity waveforms were considered abnormal when Uterine artery S/D ratio exceeds 95th percentile and Early diastolic notch in either right/ left or both uterine arteries. Umbilical artery S/D ratio,
PI exceeds 95th percentile and Absent and reverse end diastolic flow velocity in umbilical artery. Middle cerebral artery PI below 5th percentile.

(Uterine and umbilical arteries values between 90th and 95th percentile and MCA values between 5th and 10th percentile are considered as borderline83.)

These patients were followed up till delivery and details of pregnancy events, labour and delivery and neonatal outcome were noted. The abnormal pregnancy outcomes considered are IUGR, IUD, Apgar at $5 \mathrm{~min}$, NICU admission and birth weight. IUGR is defined as $<10 \mathrm{~h}$ percentile birth weight for that gestational age.

\section{RESULTS}

Table -1 : Correlation of Uterine artery Doppler with Pregnancy outcome

\begin{tabular}{|l|l|l|l|l|l|}
\hline Doppler Index & IUGR & IUD & STILL BIRTH & NICU ADMISSION & APGAR AT 1'<7 \\
\hline S/D & 17 & 1 & 1 & 11 & 6 \\
\hline Early Diastolic Notch & 28 & 2 & 2 & 21 & 17 \\
\hline
\end{tabular}

Table-2 : Correlation of Umbilical artery Doppler with pregnancy outcome

\begin{tabular}{|l|l|l|l|l|l|}
\hline Umbilical artery & IUGR & IUD & Still birth & NICU Admission & Apgar at 1'<7 \\
\hline S/D Ratio & 20 & 2 & 2 & 15 & 8 \\
\hline P.I & 15 & 3 & 4 & 9 & 6 \\
\hline AEDV/REDV & 9 & 3 & 4 & 2 & 2 \\
\hline
\end{tabular}

Table-3 : Correlation of MCA P.I with Pregnancy outcome

\begin{tabular}{|l|l|l|l|l|l|}
\hline MCA & IUGR & IUD & STILL BIRTH & NICU ADMISSION & APGAR AT 1'<7 \\
\hline P.I & 8 & 2 & 2 & 5 & 4 \\
\hline
\end{tabular}

Table -4 : Diagnostic accuracy of Doppler in predicting adverse Perinatal outcome

\begin{tabular}{|l|l|l|l|l|}
\hline Index & $\begin{array}{l}\text { Sensitivity } \\
(\%)\end{array}$ & $\begin{array}{l}\text { Specificity } \\
(\%)\end{array}$ & PPV\%. & NPV\% \\
\hline Uterine .A S/D & 68 & 44.8 & 51.5 & 61.9 \\
\hline U.A S/D & 68.9 & 48 & 60.6 & 57.1 \\
\hline U.A PI & 51.7 & 64 & 62.5 & 53.3 \\
\hline MCA PI of & 44.4 & 93.9 & 80 & 75.6 \\
\hline $\begin{array}{l}\text { PI } \\
\text { MCA/UA }\end{array}$ & $\mathbf{6 1 . 6}$ & 87.5 & 82.3 & 56.7 \\
\hline $\begin{array}{l}\text { S/D } \\
\text { MCA/UA }\end{array}$ & 61.5 & 84.2 & 71.4 \\
\hline
\end{tabular}

In this study 54 patients were included, majority was with age range $<20$ i.e. $6(11.1 \%)$, $21-25$ in $28(51.8 \%), 26-30 \%$ in $14(25.9 \%)$, and $>30$ in $6(11.1 \%)$. At the time of scan gestational age was 20 weeks in 19(35.1\%), 21 weeks in 18(33.3\%), and $>22$ weeks in 17(31.3\%). 26(48.1\%) were PRIMI gravida, $17(31.5 \%)$ were G2 and $11(20.4 \%)$ were $>$ G3. Out of 54 patients majority had vaginal delivery i.e. 34(63\%), 12 (22\%) had emergency LSCS, and 8(15\%) had elective LSCS. 26(48\%) were primipara and 28 (52\%) were multipara. Pre-eclampsia in 29(54\%), 6(11\%) had previous H/O IUGR and other complications in 19(35\%). Indication for Csection was fetal distress in $8(40 \%)$, severe pre-eclampsia in $3(15 \%)$ and others in $10(50 \%)$. Perinatal outcome was $47(87 \%)$ live birth.

Out of 54, 47 (87\%) were live and $7(13 \%)$ were still born. 26 (48\%) were preterm and 20(37\%) were term. 15(28\%) had birthweight $>2500$, 26(48\%) had 15002500, 11 (20\%) had 1000-1500. Apgar score at $1^{\prime}<7$ in 25(47\%), 27 (50\%) admitted to NICU. 20(37\%) had neonatal complications. Duration of stay $>10$ days in $15(28 \%)$.

Majority of infants developed birth asphyxia i.e. 8 followed by meconium aspiration in 6, Polycythemia, Hypocalcemia and Hypoglycemia in 2 each. Uterine artery Doppler was abnormal in 33 
patients. Out of 33, 17 patients had IUGR babies with adverse perinatal outcome. The sensitivity and specificity of abnormal uterine artery Doppler was 68\% and $44.8 \%$ respectively.

Forty patients had early diastolic notch in one or both the uterine arteries. Out of 40 ,28 patients had IUGR babies, 3 IUD, 3 still birth, 21 NICU admissions and 17 babies with Apgar $<7$ at $1 \mathrm{~min}$.

Reverse, absence or slow end diastolic flow was considered abnormal which was seen in 33 patients. Out of 33, 20 patients had IUGR babies with adverse perinatal outcome. The sensitivity and specificity of abnormal umbilical artery doppler was $68.9 \%$ and $48 \%$ respectively. Nine patients had Absent /Reversed end diastolic flow of which, 3 patients had IUD, 4 had Still births, 2 were admitted in NICU with Apgar at $1 \mathrm{~min}<7$. In Middle cerebral artery we assessed the pulsatility index and if PI is less than 1.4 we take it as low and was found in $10(18.5 \%)$ of patients. Middle cerebral artery was abnormal in 10 patients, of which 8 patients had IUGR babies with adverse perinatal outcome. The sensitivity and specificity of abnormal MCA Doppler was $44.4 \%$ and $93.9 \%$ respectively.

We also analysed the MCA/UA S.D ratio, which was abnormal in 19 patients of which 16 patients had adverse perinatal outcome. The sensitivity and specificity of abnormal MCA/UA S.D ratio was 61.5\% and $89.2 \%$ respectively. The sensitivity and specificity of abnormal MCA/UA P.I ratio was $46.6 \%$ and $87.5 \%$ respectively.

\section{DISCUSSION}

Intrauterine growth retardation and pregnancy-induced hypertension are both pathological disorders that are closely linked to the development and function of the uteroplacental and fetoplacental circulations. As a result, blood flow in the uterine and umbilical arteries is disrupted. Normal prenatal growth necessitates good foetal circulation. Significant changes in foetal and maternal vasculature occur to facilitate this.
By the 20th week of pregnancy, haemodynamic changes on the maternal side of the placenta are reflected in the Uterine artery. By 25 weeks, trophoblastic cells had penetrated the maternal spiral arteries within the inner one-third of myometrium, dissolving the spiral arteries' internal elastic lamina. As a result, the arteries dilate to their full capacity and vascular resistance is reduced to a minimum.

In the $\mathrm{PIH}$, there is insufficient invasion, resulting in an increase in spinal artery resistance. This causes blood flow in the uterine arteries to be obstructed6. A S/D ratio of more than 2.6 in the uterine arteries is deemed abnormal. Up to 26 weeks of pregnancy, the appearance of an early diastolic notch is a common occurrence. The existence of a notch after 26 weeks is a poor sign, indicating increased blood flow impedance. When the S/D ratio in the right and left uterine arteries differs by more than one, it indicates a higher risk of poor foetal outcome.

The velocimetry of the umbilical artery correlates with variations in the fetoplacental circulation's haemodynamics. The feto placental compartment develops when the number of tertiary stem villi and arterial channels increases, and the resistance in the umbilical artery lowers. Umbilical artery resistance decreases after 15 weeks of pregnancy, and the diastolic component shows in the waveform during the early second trimester. A normal S/D ratio is less than or equal to three.

First, IUGR causes a decrease in diastolic flow in the umbilical artery due to an increase in resistance in the tertiary villi's tiny arteries and arterioles. This increases the P.I. and R.I. of the umbilical artery. The diastolic flow declines as placental insufficiency progresses, eventually becomes absent, and ultimately reverses. Due to a lesser form of placental insufficiency, some foetuses have a lower diastolic velocity that remains consistent with advancing gestation and never becomes missing or reversed. 
Perinatal death is reported to be more than $40 \%$ common in foetuses with absent end diastolic flow velocity. Yoon et al7 found that the absence of end diastolic flow in the umbilical artery waveform is a strong and independent predictor of poor neonatal outcome in their investigation.

It is a low-resistance circulation that contributes for $7 \%$ of foetal cardiac output during pregnancy. According to Mark et al8, the MCA impedance changes during pregnancy. During pregnancy, it follows a parabolic pattern and does not change significantly after delivery.

The brain sparing that occurs in the impaired foetuses is shown by an increase in diastolic flow with a decrease in PI. The cerebral placental ratio is constant during pregnancy, especially after 30 weeks, according to Gramellini D et al9, who established a cut-off value of 1.08; all values less than 1 are considered abnormal. When compared to the PI of MCA alone (50 percent) for five minutes APGAR score, this ratio has a higher sensitivity (100 percent).

This study was primarily done to evaluate whether abnormal Doppler finding in second and third trimester of high risk pregnant woman is useful in predicting development of IUGR, Pre-term delivery and NICU admission.

When the fetal placental flow is severely affected there is an increased impedance to flow resulting in end diastolic flow becoming absent. With further hemodynamic compromise there will be reversal of flow in the umbilical arteries. Such a development is ominous and results in a profoundly adverse perinatal outcome10.

In our study it was seen that AEDF or REDF correlated with poor perinatal outcome with an increase in the perinatal mortality and morbidity. The sensitivity, specificity, positive predictive value (PPV) and negative predictive value (NPV) of Umbilical artery $\mathrm{PI}, \mathrm{S} / \mathrm{D}$ ratio, MCA PI,MCA /UA PI and MCA/UA S/D ratios in our study correlated with the results of the study done by B N Lakhkar et al.11.
Absent Reversed end diastolic flow in Umbilical artery was seen in 9 patients. All patients had poor perinatal outcome with 2 IUGR, 3 Intra uterine deaths, 4 Still births and 2 babies had NICU admission with Apgar score at $1 \mathrm{~min}<7$. This correlated with the studies done by Yoon et al7, Battaglia et al12 and BN Lakhkar et al11.

We found that in predicting adverse neonatal outcome, the ratio of PI of MCA/UA is more sensitive than MCA PI alone which is similar to study by $\mathrm{BN}$ Lakhkar et al11 but in contrast to Shahino Bano et al13. This may be due to ethnic variations in the study population. The MCA/UA pulsatality index ratio may be more useful in predicting perinatal outcome because it includes data on both placental health and fetal responsiveness. The $\mathrm{C} / \mathrm{U}$ ratio was computed by Gramellini et al.9 and shown to be stable in the last 10 weeks of pregnancy. They've also demonstrated that it has a higher diagnostic accuracy than either vessel's PI alone.

Our study is comparable to Gramellini et al9 .Rozeta et al14 studied in 738 patients and our study is on 54 patients the difference in results with study of Rozeta et al14 may be due to smaller study population and also may be due to ethnic variations. The MCA/UA P.I. was compared to the Sharma DD et al15 study. Sixty singleton pregnancies identified with IUGR after 30 weeks of pregnancy were assessed and tracked clinically as well as with Doppler findings till birth, according to Sharma et al15. The prognostic efficiency of umbilical artery and middle cerebral artery waveforms was investigated utilizing percentages (sensitivity, specificity, positive and negative predictive values).

They discovered that MCA PI had the best sensitivity and specificity (78 percent and 93 percent, respectively) as an indicator for the level of fetal impairment in IUGR, however ratios like MCA PI/UA PI and UA SD (>3) were also highly specific predictors of poor perinatal outcome. The absence or reversal of end diastolic flow in the umbilical artery is linked to a poor 
perinatal outcome in terms of mortality. In our study it is the cerebro placental ratio which has higher specificity, PPV, NPV as compared to their study which shows MCA PI has higher sensitivity, Specificity, PPV, NPV.

\section{CONCLUSION}

Doppler has proven to be more sensitive than other methods of fetal monitoring in recognizing fetal compromises early and assisting in the proper time of delivery. The Umbilical artery is the first to change in doppler parameters, followed by the middle cerebral and other peripheral arteries. As a result, growth-restricted fetuses are followed using a Doppler investigation of fetal circulation, allowing for optimal delivery time. Neonatal morbidity can be predicted using fetal Doppler indices, particularly ratios that include readings from the cerebral circulation. As a result, the use of Doppler delivers information that is not readily available from more traditional fetal wellbeing exams.

\section{Acknowledgement: None}

\section{Conflict of Interest: None}

\section{Source of Funding: None}

\section{REFERENCES}

1. Alan, H.D and N. Lauren. Text Book of current diagnosis and Treatment, Obstetrics and Gynecology. Tenth edition.

2. Asim kuorjak,Frank A Cherven. Textbook of ultrasound of gynecology and obstetric 1st ed.2006.

3. John, C,H. Obstetric Ultrasound : Artistry in Practice. 1st ed, 2008, U.S:John Wiley\&Sons.

4. Arey LB. Developmental Anatomy: A text book and laboratory manual of Embryology. 7th ed. Philadelphia 2011.

5. Jane A Bates et al. Differentiation of growth retarded from normally grown fetuses and prediction of intrauterine growth retardation using Doppler ultrasound. BJOG.1996;103(7) ;670675.

6. Fleischer A, Schulman H, Farmakides G et al. Uterine artery Doppler velocimetry in pregnant women with hypertension. Am J Obstet Gynecol. 1986 Apr; 154(4):806-13.

7. Yoon BH, Lee CM, Kim SW. An abnormal umbilical artery waveform: a strong and independent predictor of adverse perinatal outcome in patients with preeclampsia. Am J Obstet Gynecol. 1994 Sep;171(3):713-21.

8. Mari G, Deter RL. Middle cerebral artery flow velocity waveforms in normal and small-for-gestational-age fetuses. Am J Obstet Gynecol. 1992 Apr; 166(4): 1262-70.

9. Gramellini D et al. Cerebral-umbilical Doppler ratio as a predictor of adverse perinatal outcome. Obstet Gynecol. 1992 Mar; 79(3):416-20.

10. Bhatt CJ, Arora J, Shah MS. Role of color Doppler in pregnancy induced hypertension (a study of 100 cases). Indian J Radiol Imaging. 2003;13:41720.

11. BN Lakhkar, KV Rajagopal, PT Gourisankar. Doppler predilection of adverse perinatal outcome in $\mathrm{PIH}$ and IUGR. Ind $\mathrm{j}$ radiol imag. 2006; 16(1):109-116.

12. Battaglia $C$ et al. Absent or reverse \& diastolic flow in umbilical artery and severe intrauterine growth retardation. Acta Obstet Gynecol. 1993; 72:167171.

13. Shahina Bano et al. Color Doppler evaluation of cerebral umbilical pulsatility ratio and its usefulness in the diagnosis of intra uterine growth restriction and prediction of adverse perinatal outcome. IJRI.2010 feb ;20(1):20-25.

14. Shahinaj R, Manoku N, Kroi E, Tasha I. The value of the middle cerebral to umbilical artery Doppler ratio in the prediction of neonatal outcome in patient with preeclampsia and 
gestational hypertension. J Prenat Med. 2010 Apr-Jun; 4(2):17-21.

15. Sharma DD et al. Clinical study of IUGR cases and correlation of Doppler parameters with perinatal outcome. Int $\mathrm{J}$ Reprod Contracept Obstet Gynecol. 2016 Dec;5(12):4290-4296.
How to cite this article: Gattani Chandrashekhar M, Pradeep R. Role of ultrasonography in screening high risk mothers for detection of IUGR. International Journal of Research and Review. 2021; 8(11): 106-112. DOI: https://doi.org/10.52403/ijrr.20211114 